\title{
A Novel AND Single ChIP TRI-BAND LOW- NOISE AMPLIFIER FOR WLAN, WIFI AND WiMAX RECEIVERS
}

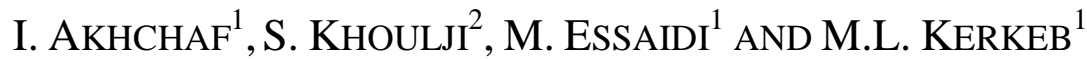 \\ ${ }^{1}$ Information and Telecommunications Systems Laboratory, Physics Department, Faculty \\ of Sciences, Abdelmalek Essaadi University Tetuan, Morocco \\ akh.ibtissam@gmail.com- essaaidi@ieee.org- kerkebml@uae.ma- \\ ${ }^{2}$ Department of Statistics and Informatics, Faculty polydisciplinary of Tetouan, \\ Abdelmalek Essaadi University Tetuan, Morocco \\ khouljisamira@gmail.com
}

\begin{abstract}
In first stage of each microwave receiver there is Low Noise Amplifier (LNA) circuit, and this stage has important rule in quality factor of the receiver. The design of a LNA in Radio Frequency (RF) circuit requires the trade-off many importance characteristics such as gain, Noise Figure (NF), stability, power consumption and complexity. This situation forces designers to make choices in the design of RF circuits. In this paper the aim is to design and simulate a novel and single Chip consists of a tri-band low noise amplifier (LNA) operating simultaneously at three frequency bands $1.9 \mathrm{GHz}, 2.4 \mathrm{GHz}$ and $5 \mathrm{GHz}$ for WLAN, WiFi and WiMax receivers, is designed and presented in this paper. It can be used in many applications of wireless communication (GSM, Zigbee, Bluetooth, Wi-Fi, HiperLAN, UWB, etc ...) for $3 G$ and $4 G$ networks. The proposed circuit is based on two amplifiers, dual-band and simple band, with transistor GaAs FET type ATF 10136 that has a better performance, successful integration of feasibility and a low price compared with other technologies. Simulations of the operation of the amplifier were performed with the software Agilent Advanced Design System (ADS) and Ansoft software and performance of the amplifier were recorded and analyzed. A single stage LNA has successfully been designed with $29.3 \mathrm{~dB}, 24.56 \mathrm{~dB}$ and $11.93 \mathrm{~dB}$ with noise figure of $0.44 \mathrm{~dB}, 0.49 \mathrm{~dB}$ and $4.42 \mathrm{~dB}$ respectively at three frequency bands $1.9 \mathrm{GHz}, 2.5 \mathrm{GHz}$ and $5 \mathrm{GHz}$.
\end{abstract}

\section{KEYWORDS}

WLAN, WiFi and WiMax receivers, Networks and Telecommunications, System Radio Frequency $(R F)$, wireless communication, GaAs FET ATF 10136, Low Noise Amplifiers (LNA), band-pass filter (BPF), dual bandamplifiers, tri-band amplifiers, Microwave devices, Advanced Design System (ADS), Ansoft .

\section{INTRODUCTION}

In recent years, the frequency bands used in the telecommunications sector have been increasing due to the saturation of the traditional bands, leading always to explore the frequency bands of higher and higher and not used. As a result, techniques and architectures currently used DOI : $10.5121 /$ ijcsit.2012.4605 
in radio frequency will be improved to adapt to new bands and new features of telecommunications. Wireless telephony uses different frequency bands $(900 \mathrm{MHz}, 1800 \mathrm{MHz}$, $1900 \mathrm{MHz}, 2200 \mathrm{MHz}$...), hence most mobile phones on the market nowadays offer to cover these bands with tri-band or quad-band mobile phones, which requires the multiplication of channels of receive and transmit radio frequency to treat each of these frequencies. This increases costs and causes usability issues since you need more space to perform the multitude of channels [2-7].

In order to address these problems, we propose to develop circuits capable of operating over a frequency band or another, depending on the choice of the user. Indeed, the realization of this concept can be used to design a single RF receiver chain can process according to the choice of the user number of telecommunications norms. The microwave devices should be more rapid and must cover wide frequency bands in order to monitor progress in the field of mobile telephony with its multitude of protocols (GSM, CDMA, CDMA2000 etc ....) as well as evolution and development of local networks. The list of standards for the tri-band LNA in this work is shown in Table I [4].

Table 1: Frequency Bands

\begin{tabular}{|c|c|c|}
\hline Frequency Band & Wireless standards & $\begin{array}{c}\text { Frequency Spectrum } \\
\text { (MHz) }\end{array}$ \\
\hline \multirow{2}{*}{$\mathbf{1}$} & GSM & $935 \sim 960$ \\
\hline \multirow{4}{*}{$\mathbf{4}$} & DCS-1800 & $1805 \sim 1850$ \\
\cline { 2 - 3 } & DECT & $1881 \sim 1897$ \\
\cline { 2 - 3 } & PCS-1900 & $1930 \sim 1960$ \\
\cline { 2 - 3 } & WCDMA & $2110 \sim 2170$ \\
\cline { 2 - 3 } & WLAN (802.11b/g) & $2400 \sim 2483$ \\
\hline \multirow{3}{*}{3} & Bleutooth (802.11FH) & $2400 \sim 2483$ \\
\cline { 2 - 3 } & WiMax (802.16d-2004) & $2000 \sim 11000$ \\
\cline { 2 - 3 } & WiMax (802.16e-2005)for Fixed & $2000 \sim 11000$ \\
\hline \multirow{4}{*}{4} & WiFi (802.16e-2005) for Mobile & $2000 \sim 6000$ \\
\cline { 2 - 3 } & WiFi (802.11a) & $5150 \sim 5825$ \\
\cline { 2 - 3 } & WiFi (802.11ac) & $5150 \sim 5825$ \\
\cline { 2 - 3 } & WiFi (802.11ad) & $5150 \sim 5825$ \\
\hline \multirow{2}{*}{$\mathbf{5}$} & WiFi (802.16a) & $10000 \sim 66000$ \\
\hline
\end{tabular}

The standards are grouped into bands based on their carrier frequencies to determine the number of notches required and their corresponding inter-band notch frequencies.

As the wireless communication system becomes mature and widespread, the requirement of a low noise amplifier (LNA) for the system has become a lot more sophisticated. However, low noise amplifier is one of the most critical building blocks in modern integrated radio frequency (RF) transceivers for wireless communications. The LNA is a very important device which is placed in front of receiver and determines noise 
figure of the whole receiver. Main performance parameters of the LNA are the gain, noise figure, and linearity. Among them, the gain and noise figure directly affect the noise figure of the whole receiver and the previous researches of the LNA are focused on the gain and noise figure.

For low cost and high integration consideration, the transistor FET process has become one of the most popular technologies to provide excellent integration with other baseband blocks. Besides, modern wireless applications necessitate communication systems with more functionality and flexibility. Therefore, multi-standard RF transceivers integrated using transistor FET processes are predicted to play a critical role in the future wireless communication system [18-20]. If we can combine two or more RF standards into one transceiver, it will reduce cost and improve integration in advanced. However, a suitable LNA must be designed and realized before implementing a multi-standard receiver. And two ways can approach this goal, wideband or multi-band structure [4-6].

Recently, many researches [11-16] about dual-band LNA have been studied and reported. However, the concurrent triple band LNA is rarely cited and studied. In this work, a new fully integrated concurrent triple-band LNA is first proposed that is capable of simultaneous operation at all three different frequency band $(1.9 \mathrm{GHz}, 2.5 \mathrm{GHz}$, and $5 \mathrm{GHz})$ without dissipating triple as much power or a significant increase in cost and footprint. Our study focuses on the design and implementation of trial band LNA based on two amplifiers, dual band and single band with a single transistor FET that offers good performance and high integration feasibility, to obtain good return loss, low noise and high gain simultaneously. To provide some background, section II review a concurrent tri-band LNA architecture. In section III, we present our analysis and concepts of designing a fully integrated concurrent triple-band LNA in advanced and we show its post simulation performance. In Section IV, we conclude for this paper.

\section{TRANSISTOR ATF 10136 TECHNOLOGIES (GAAS FET)}

The proposed circuit was implemented using Transistor ATF 10136 technologies that is a high performance gallium arsenide Schottky-barriergate field (GaAs FET) effect transistor housed in a cost effective microstrip package and its premium noise figure makes this device appropriate for use in the first stage of low noise amplifiers operating in the $0.5-12 \mathrm{GHz}$ frequency range and it operates under a $5 \mathrm{~V}$ supply. This GaAs FET device has a nominal 0.3 micron gate length using airbridge interconnects between drain fingers. Total gate periphery is 500 microns. Proven gold based metallization systems and nitride passivation assure a rugged, reliable device $[17,20]$.

\subsection{Gallium arsenide field-effect transistor (GaAs FET)}

A gallium arsenide field-effect transistor (GaAsFET) is a specialized type of field-effect transistor ( FET ) that is used in amplifier circuits at very-high, ultra-high, and microwave radio frequencies. This spans the electromagnetic radiation spectrum from approximately $30 \mathrm{MHz}$ up to the infrared band. The GaAs FET is known for its sensitivity, and especially for the fact that it generates very little internal noise. This is because gallium arsenide has exceptional carrier mobility. The electrons and holes move through the semiconductor material easily and fast. The GaAs FET is a depletion-mode device. This means that it conducts when no voltage is applied to the control electrode (gate), and when a voltage appears at the gate, the channel conductivity decreases. 
In weak-signal wireless communications and broadcast reception, GaAsFET devices perform better than most other types of FET. Some types of GaAsFET are used as radio-frequency (RF ) power amplifiers. GaAsFETs are employed in space communications, in radio astronomy, and in experiments conducted by amateur radio operators [17].

\subsection{Field-effect transistor (FET)}

A field-effect transistor (FET) is a type of transistor commonly used for weak-signal amplification (for example, for amplifying wireless signals). The device can amplify analog or digital signals. It can also switch DC or function as an oscillator.

In the FET, current flows along a semiconductor path called the channel. At one end of the channel, there is an electrode called the source. At the other end of the channel, there is an electrode called the drain. The physical diameter of the channel is fixed, but its effective electrical diameter can be varied by the application of a voltage to a control electrode called the gate. The conductivity of the FET depends, at any given instant in time, on the electrical diameter of the channel. A small change in gate voltage can cause a large variation in the current from the source to the drain. This is how the FET amplifies signals.

Field-effect transistors exist in two major classifications. These are known as the junction FET (JFET) and the metal-oxide- semiconductor FET (MOSFET).

The junction FET has a channel consisting of N-type semiconductor (N-channel) or P-type semiconductor (P-channel) material; the gate is made of the opposite semiconductor type. In Ptype material, electric charges are carried mainly in the form of electron deficiencies called holes. In N-type material, the charge carriers are primarily electrons. In a JFET, the junction is the boundary between the channel and the gate. Normally, this P-N junction is reverse-biased (a DC voltage is applied to it) so that no current flows between the channel and the gate. However, under some conditions there is a small current through the junction during part of the input signal cycle.

In the MOSFET, the channel can be either N-type or P-type semiconductor. The gate electrode is a piece of metal whose surface is oxidized. The oxide layer electrically insulates the gate from the channel. For this reason, the MOSFET was originally called theinsulated-gate FET (IGFET), but this term is now rarely used. Because the oxide layer acts as a dielectric, there is essentially never any current between the gate and the channel during any part of the signal cycle. This gives the MOSFET an extremely large inputimpedance. Because the oxide layer is extremely thin, the MOSFET is susceptible to destruction by electrostatic charges. Special precautions are necessary when handling or transporting MOS devices.

The FET has some advantages and some disadvantages relative to the bipolar transistor. Fieldeffect transistors are preferred for weak-signal work, for example in wirelesscommunications and broadcast receivers. They are also preferred in circuits and systems requiring high impedance. The FET is not, in general, used for high-power amplification, such as is required in large wireless communications and broadcast transmitters. 
Field-effect transistors are fabricated onto silicon integrated circuit (IC) chips. A single IC can contain many thousands of FETs, along with other components such as resistors, capacitors, and diodes $[18,20]$.

\section{Circuit Design}

\subsection{A review of dual band LNA}

Similar to the single-band LNA [2], being the first active element of the receiver chain, the noise figure (NF) of a dual-band LNA [7] also plays a significant role in the overall NF of the dualband receiver. Before explaining the design details of concurrent triple-band LNA, it is helpful to review some basic design guidance and concurrent dual-band LNA architecture. The structure of concurrent dual-band LNA is shown in Figure. 1.

In a common source configuration, inductive degeneration is used to generate the real part needed to match the LNA input to the preceding antenna or filter. Inductive degeneration can also enhance the output signal-to-noise ratio (SNR) of the receiver. The inductive feedback moves the source impedance for optimum NF toward the optimum power match with a minor increase in the minimum NF. Cascade configuration can be used to enhance stability, reverse isolation of the amplifier and reduce Miller effect [2-5].

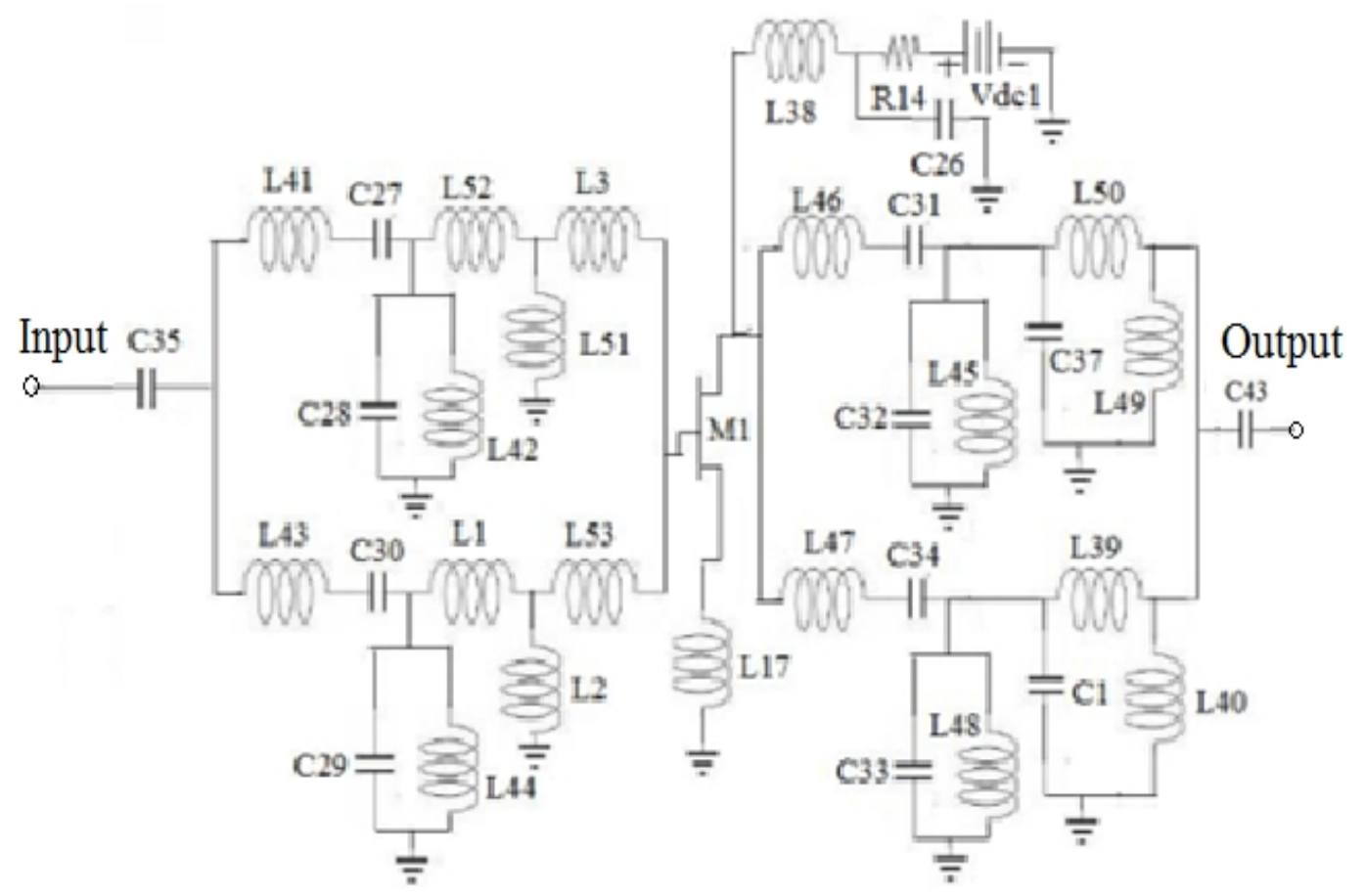

Figure. 1 Concurrent dual-band LNA with only one transistor 


\subsection{Tri- Band LNA architecture}

This section, we propose new triple-band LNA architecture based on above dual-band LNA for cellular Transceiver in the Wireless Applications. As shown in Figure. 2, a two-stage topology with bias-current reuse technique has been used to simultaneously achieve high gain and good matching without large amount of power consumption at all three desired band. The proposed circuit was implemented using Transistor ATF 10136 technologies. We will show its post simulation results to demonstrate this good performance.

The first stage consisted of only one transistor M1 is similar to dual-band LNA architecture, which adopted a source inductive degeneration cascade configuration. The second stage (M2) is isolated from the first one by bypass capacitor and RF signals are fed into the second stage through coupling capacitor. Based on the characteristics of LC (Inductor-Capacitor) series resonance (short circuit) and parallel resonance (open circuit), we can simultaneously design and realize input and output matched to about 50ohm. Similar to dual-band LNA [10-13], output matching network will provide two zeros between each band. Large image rejection in excess of that of the single sideband receiver is achieved through diligent frequency planning and proper usage of stop-band attenuation. In particular, we introduce some feedback and coupling capacitors to adjust bandwidth of desired bands and spacing between each band.

If the noise factor is too large, this is stabilizing resistance placed in input (the thermal noise is amplified by the transistor which degrades the high NF). We can then use resonant circuits in the sensitive circuit of frequency stabilizing resistances.

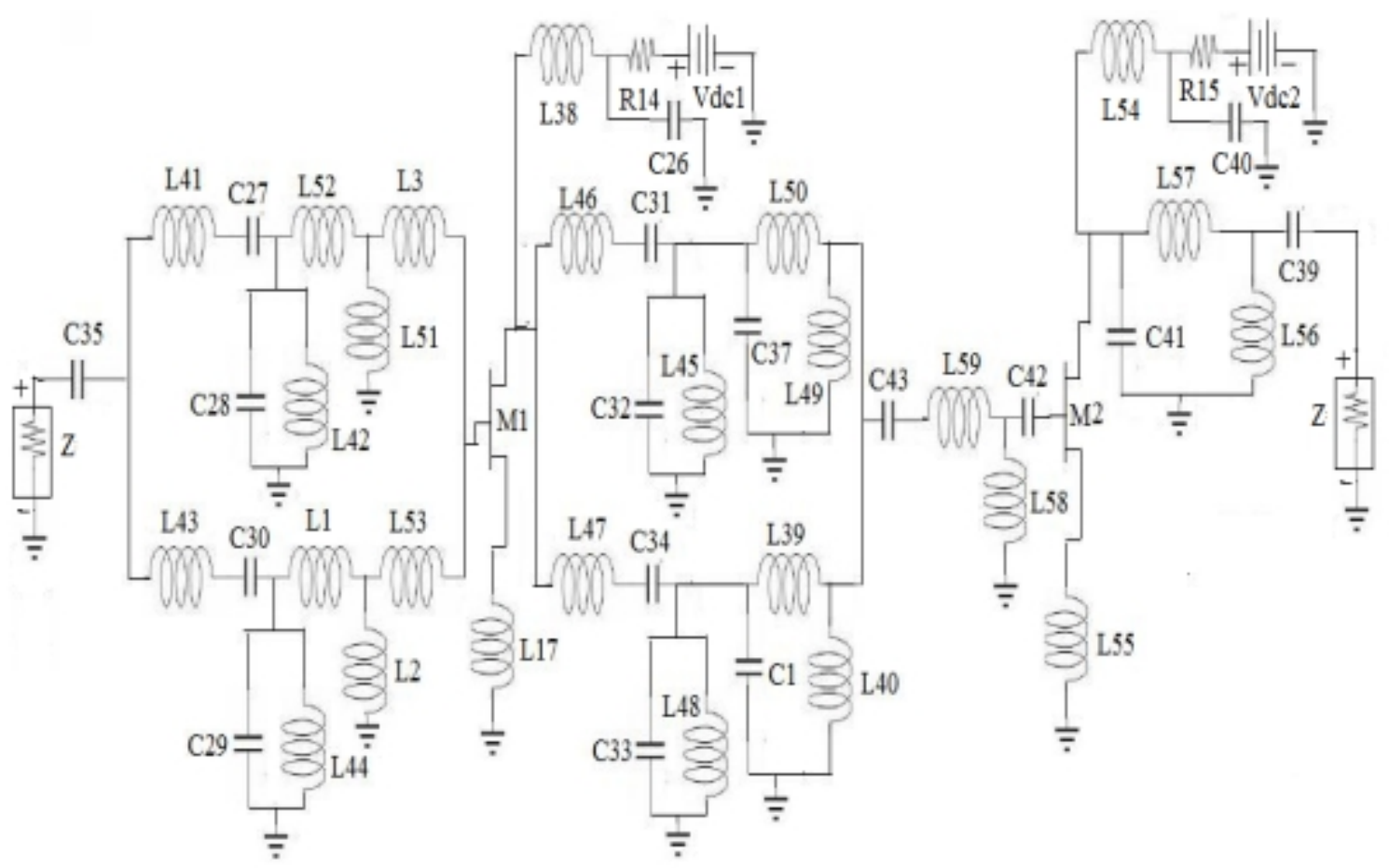

Figure 2. Schematic of the proposed tri-band LNA for Cellular Transceiver in the Wireless Applications 


\section{Stability Consideration}

The stability of an LNA amplifier, or its resistance to oscillate, is a very important consideration in a design and can be determined from the $\mathrm{S}$ parameters, the matching networks, and the terminations. The stability of an LNA amplifier circuit can also be studied with the help of the Rolett factor. This is more useful when viewing larger frequency spectral [17].

$\mathrm{k}=\frac{1-\left\lfloor\mathrm{S}_{11}\right\rfloor^{2}-\left\lfloor\mathrm{S}_{22}\right\rfloor^{2}+\lfloor\Delta]^{2}}{2 \mathrm{~S}_{12}|| \mathrm{S}_{21} \mid}>1$

Where: $|\Delta|=\left|S_{11} S_{22}-S_{12} S_{21}\right|$

To design an unconditionally stable LNA amplifier circuit, which implies that the amplifier remains stable within the entire domain of the Smith Chart at the selected frequency and the given bias conditions, the following conditions must be met.

$\left|S_{11}\right|<1$ and $\left|S_{22}\right|<1$

As well as: $\mathrm{k}>1$ and $|\Delta|<1$

\section{Noise Figure Considerations}

Besides stability and gain, another important design parameter in receiver design, which is the measure of receiver noise, is the noise factor $\mathrm{F}$ (also known as NF, when expressed in decibels) consideration for a microwave amplifier is its noise figure. In receiver applications, it is often required to have a preamplifier with as low a noise figure as possible, as the first stage of a receiver front end has the dominant effect on the noise performance of the overall system. The definition of the noise factor of any transducer (e.g., LNA, mixer, filter, etc.) given by [1] is:

$\mathrm{F}=\frac{\left({ }^{\left.\mathrm{P}_{\text {Signal }} / \mathrm{P}_{\text {Bruit }}\right)_{\text {Sortie }}}\right.}{\left({ }^{\left.\mathrm{P}_{\text {Signal }} / \mathrm{P}_{\text {Bruit }}\right)_{\text {Entrés }}}\right.}$

The value of the noise figure NF characterized by:

$\mathrm{NF}=10 \cdot \log _{10}(\mathrm{~F})$

\section{Matching Network}

The impedance matching network is lossless and is placed between the input source and the device. The need for matching network arises because amplifiers, in order to deliver maximum power to a losd, or to rform in a certain desired way must be properly terminated at both the input and the output ports. The impedance matching networks can be either designed mathematically or graphically with the aid of Smith Chart. Several types of matching networks are available, but the one used in this design is open single stubs whose length is found by matching done using smith chart manually [17]. 
This LNA circuit was the adaptation input and output by the Smith chart [9], and to lock the frequency, we used the LC filter to filter the signal. This amplifier is biased with a voltage Vds $=5 \mathrm{~V}$, and a current Ids $=2.5 \mathrm{~mA}$. Simulations of the operation of the amplifier were performed with the ADS software and the performance of the amplifier were recorded and analyzed. In next section, through ADS software and Ansoft software post simulation results we will demonstrate that this newly proposed circuit topology will meet our desired specification using these design consideration.

\section{RESULTS AND DISCUSSION}

The ADS software post simulation performance including all layouts extracted parasitic are shown in Figures 3-5. The results of these simulations have shown that the S-parameters have three peaks, $1.9 \mathrm{GHz}, 2 \mathrm{GHz}$ and $5 \mathrm{GHz}$. Figure 3 shows the noise figure (NF), Figure 4 shows the input and output matching (S11 and S22) and Figure 5 shows the power gain (S21) and reverse isolation (S12). The LNA exhibits input matching to 50ohm with $\mathrm{S} 11$ of $-9.34 \mathrm{~dB}$ at $1.9 \mathrm{GHz},-9.5 \mathrm{~dB}$ at $2.5 \mathrm{GHz}$ and $-23.5 \mathrm{~dB}$ at $5 . \mathrm{GHz}$, as well as output matching to $50 \mathrm{ohm}$ with $\mathrm{S} 22$ of $-5.5 \mathrm{~dB},-5.9 \mathrm{~dB}$ and $-3.81 \mathrm{~dB}$ respectively. And it provides forward gain (S21) of $29.13 \mathrm{~dB}$, $24.56 \mathrm{~dB}$ and $11.93 \mathrm{~dB}$ with noise figure of $0.44 \mathrm{~dB}, 0.49 \mathrm{~dB}$ and $4.42 \mathrm{~dB}$ respectively. The LNA circuit draws a dc current of $2.5 \mathrm{~mA}$ from a $5 \mathrm{~V}$ supply voltage. All the simulated results agree well because of the accuracy in device modeling and full-wave electromagnetic simulations.

The performance parameters for different carrier frequencies and its comparison with other works are summarized in Table II. Our design has achieved much higher gain than the other designs at the cost of higher power.

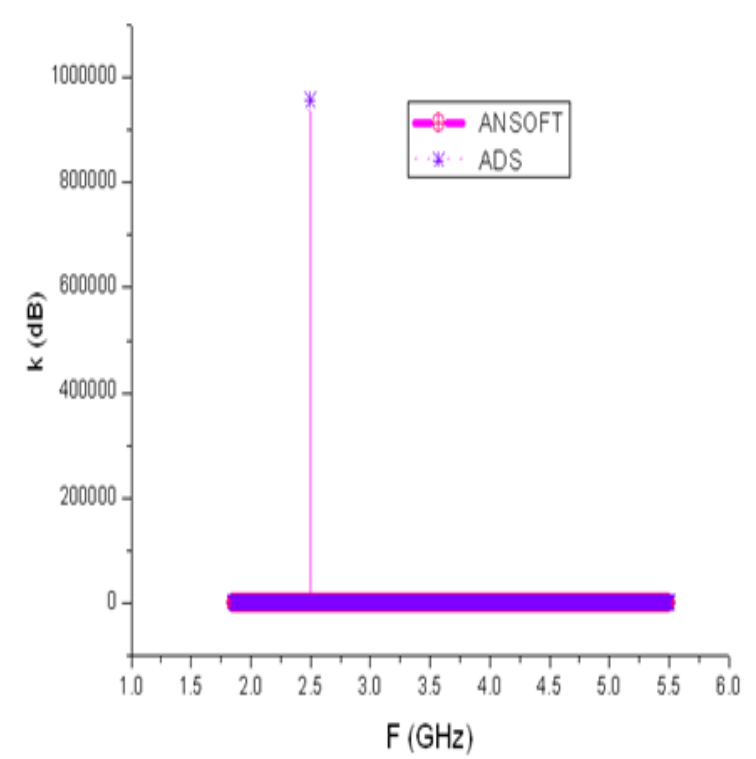

(a)

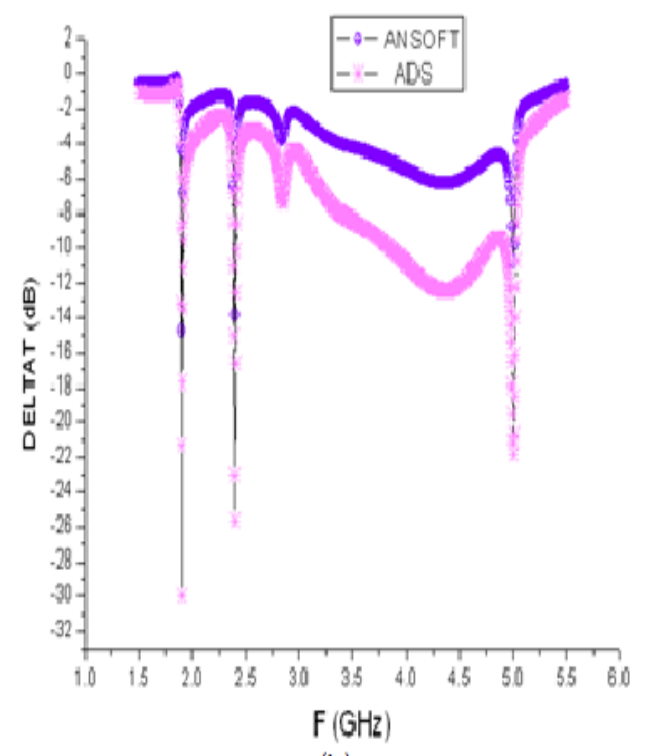

(b)

Figure 3. Stability of the proposed tri-bande LNA. (a) Rollet's factor. (b) Absolute value of delta $(\Delta<1)$. 
International Journal of Computer Science \& Information Technology (IJCSIT) Vol 4, No 6, December 2012

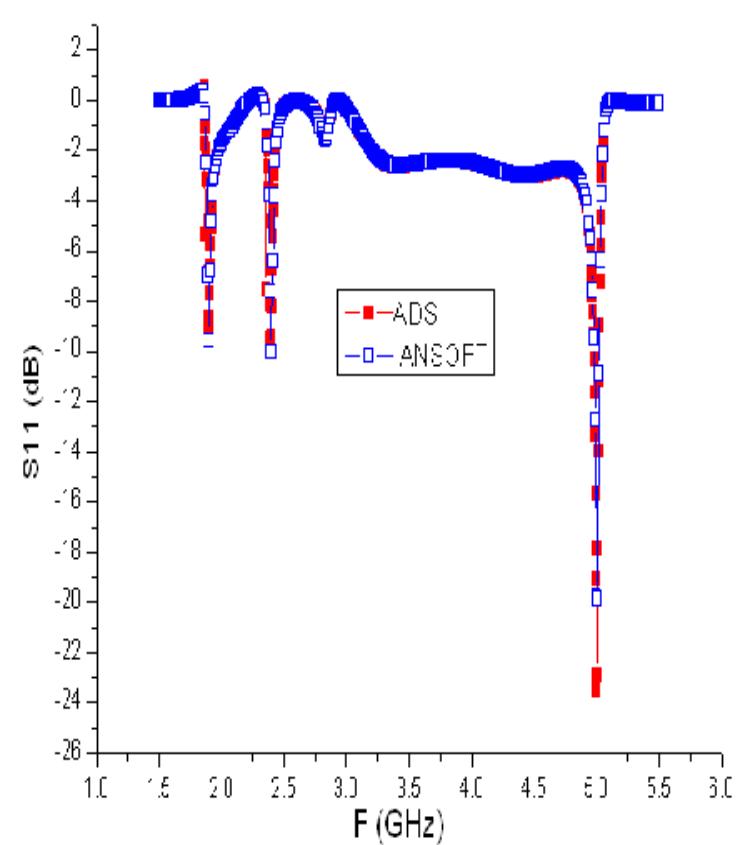

(a)

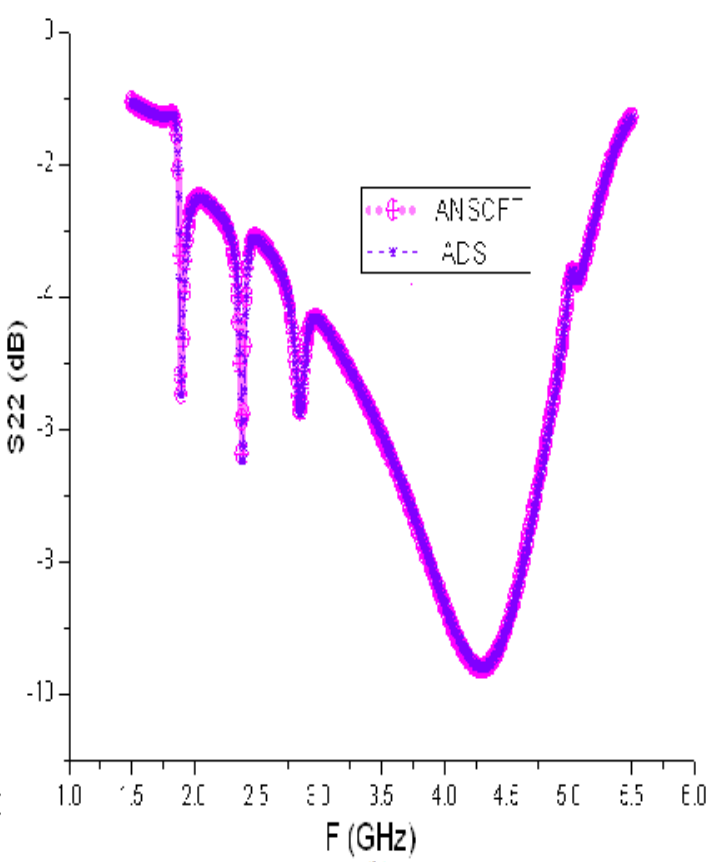

(b)

Figure 4. Input and output reflection coefficient of the proposed tri-bande LNA.

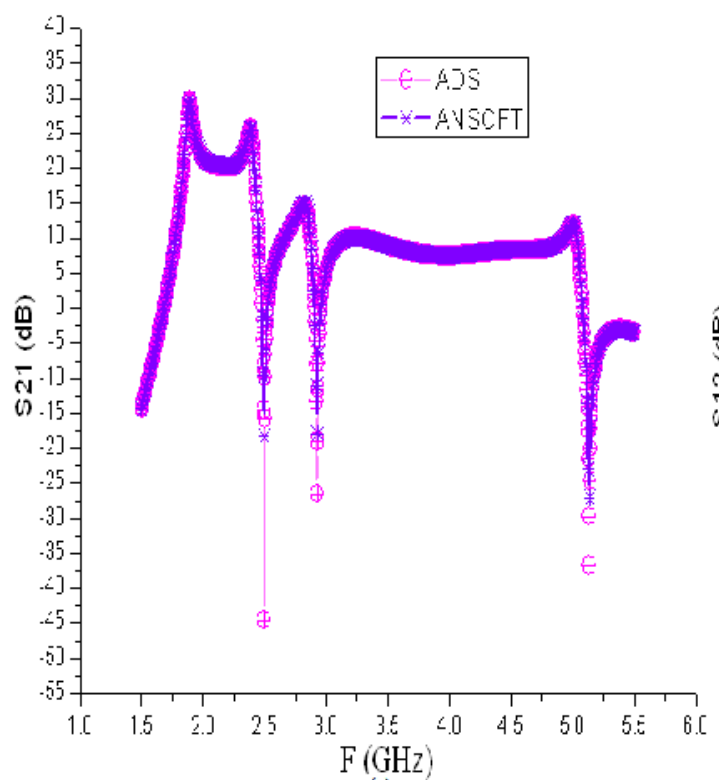

(a)

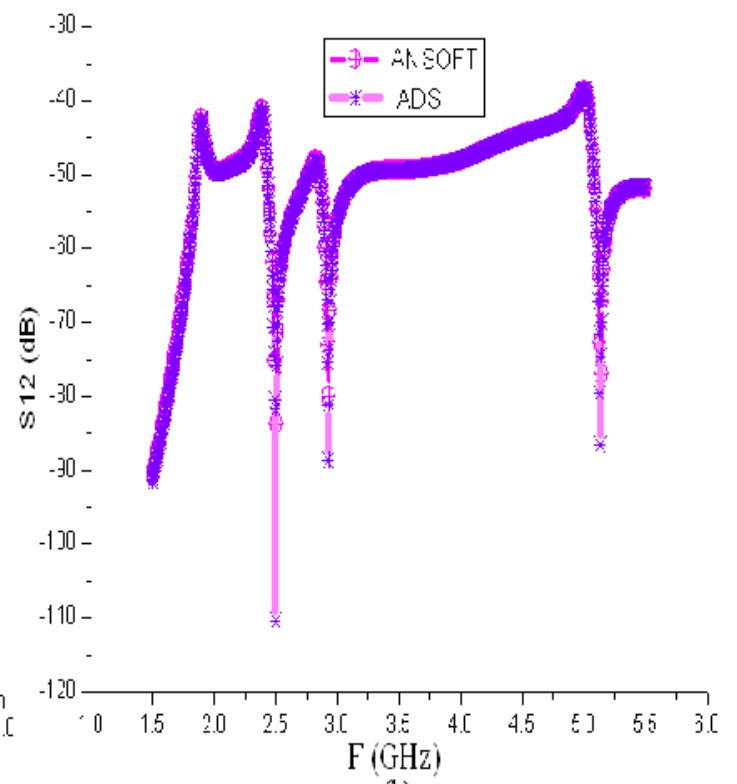

(b)

Figure 5. Gain and reverse isolation of the proposed tri-bande LNA. 
International Journal of Computer Science \& Information Technology (IJCSIT) Vol 4, No 6, December 2012

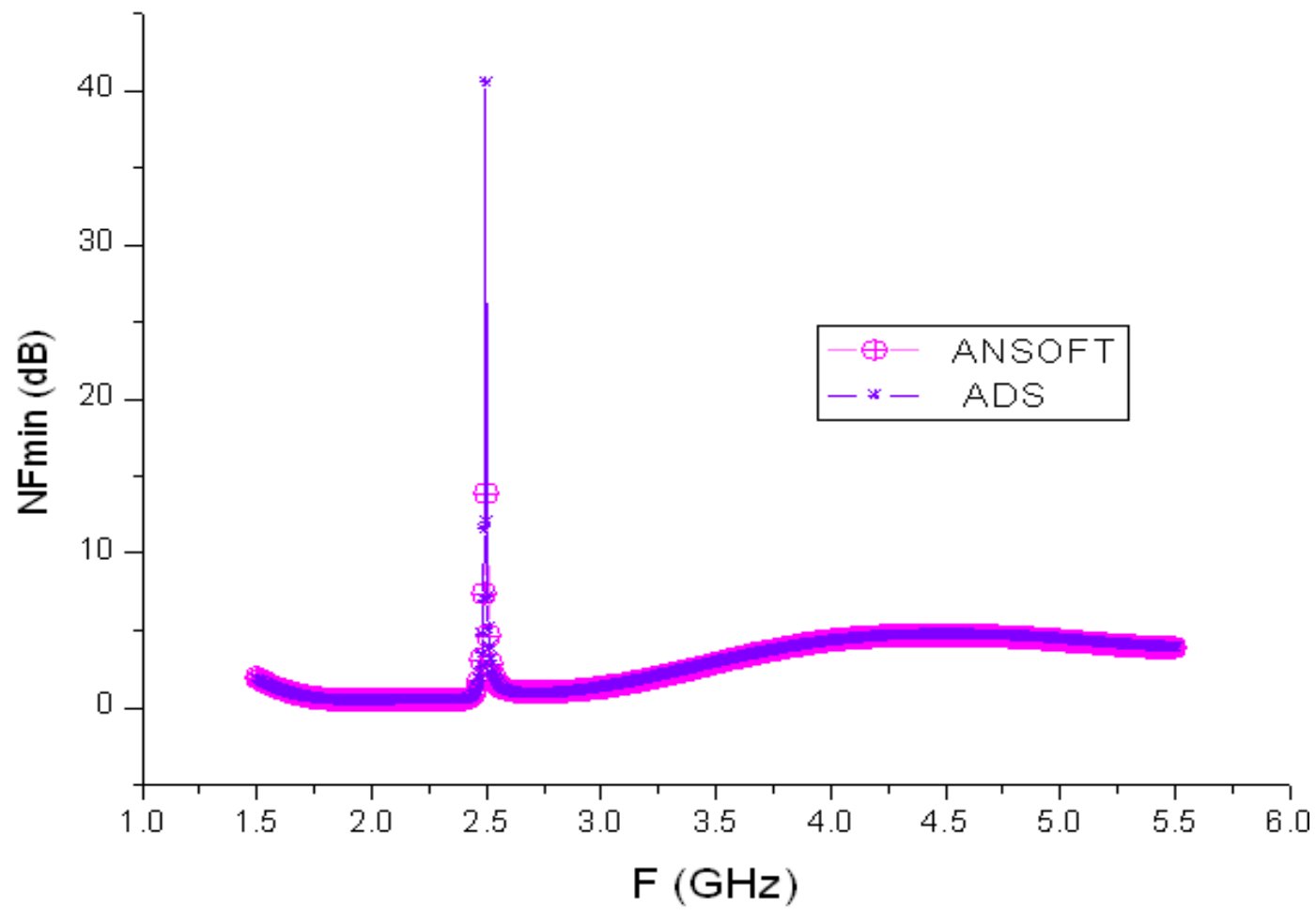

Figure 6. Noise figure of the proposed tri-bande LNA

Table II: Performance Summary and Comparison of present work and previously reported works

\begin{tabular}{|c|c|c|c|c|c|c|c|c|}
\hline Ref. & Technology & Frequency $[\mathrm{GH}]$ & $\mathrm{S} 21[\mathrm{~dB}]$ & $\mathrm{S} 12[\mathrm{~dB}]$ & $\operatorname{Sll}[\mathrm{dB}]$ & $\mathrm{S} 22[\mathrm{~dB}]$ & $\operatorname{NFmin}[\mathrm{dB}]$ & DC supply \\
\hline \multirow{3}{*}{ [2] } & \multirow[t]{3}{*}{ CMOS } & 1.8 & 10,1 & -67 & $-10,6$ & $-15,5$ & 3,69 & \multirow{3}{*}{$\begin{array}{c}15,7 \mathrm{~mA}- \\
2,5 \mathrm{~V}\end{array}$} \\
\hline & & 2.45 & 10,8 & $-62,5$ & $-10,4$ & $-12,5$ & 4,75 & \\
\hline & & 5,25 & 11,8 & $-54,5$ & $-19,9$ & -12 & 6,36 & \\
\hline \multirow{3}{*}{ [6] } & \multirow[t]{3}{*}{ CMOS } & 2.4 & 11,79 & $-56,4$ & $-10,37$ & $-12,47$ & 3,84 & \multirow[t]{3}{*}{$\begin{array}{c}10 \mathrm{~mA}- \\
1,4 \mathrm{~V}\end{array}$} \\
\hline & & 3.5 & 11,7 & $-51,3$ & $-10,41$ & $-12,17$ & 4,03 & \\
\hline & & 5,2 & 10,03 & $-45,5$ & $-13,56$ & $-19,32$ & 3,73 & \\
\hline \multirow{3}{*}{ [16] } & \multirow{3}{*}{ GaAs Phemt } & 2.4 & 22,4 & $-39,4$ & $-14,7$ & $-22,2$ & 0,9 & \multirow[t]{3}{*}{$20 \mathrm{~mA}-2 \mathrm{~V}$} \\
\hline & & 3.5 & 21,1 & $-38,5$ & $-16,2$ & $-31,3$ & 1,2 & \\
\hline & & 5,5 & 20,7 & $-36,5$ & $-13,6$ & $-13,7$ & 1,3 & \\
\hline \multirow{3}{*}{$\begin{array}{l}\text { This } \\
\text { Work }\end{array}$} & \multirow{3}{*}{ ATF 10136} & 1,9 & 29,13 & $-43,02$ & $-9,34$ & $-5,5$ & 0,44 & \multirow[t]{3}{*}{$2,5 \mathrm{~mA}-5 \mathrm{~V}$} \\
\hline & & 2,4 & 24,56 & $-42,32$ & $-9,5$ & $-5,9$ & 0,49 & \\
\hline & & 5 & 11,93 & $-38,56$ & $-23,5$ & $-3,81$ & 4,42 & \\
\hline
\end{tabular}


International Journal of Computer Science \& Information Technology (IJCSIT) Vol 4, No 6, December 2012

\section{Conclusions}

Amplification is one of the most basic and prevalent microwave circuit functions in modern RF and microwave systems. Microwave transistor amplifiers are rugged, low cost, reliable and can easily be integrated in both hybrid and monolithic integrated circuitry. Transistor amplifiers can be used at frequencies in excess of $100 \mathrm{GHz}$ in a wide range of applications requiring small size, low noise figure, broad bandwidth and low to medium power capacity. The design techniques used for FET amplifiers employ the full range of concepts developed in the study of microwave transmission lines, two-port networks and Smith chart presentation. Our discussion of transistor amplifier design will rely on the terminal characteristics of transistors as represented by $\mathrm{S}$ parameters. To amplify the received signal in a microwave system, a low noise amplifier (LNA) is required. Because any noise injected by components in a system is amplified by later gain stages along with the signal, it is essential that the signal be amplified early in the receiver chain while adding as little noise as possible. The goal of this is to design an LNA with lowest noise figure possible, with gain as high as possible for the given FET and information.

This work investigates a novel and single Chip circuit topology of tri-band low noise amplifier (LNA) for Cellular Transceiver in the Wireless applications. The design utilizes one highperformance low noise GaAs FET transistor. It has been implemented using transistors FET ATF 10136 technology. The proposed tri-bande LNA has been used to simultaneously achieve high gain and good matching without large amount of power consumption at all three desired band $1.9 \mathrm{GHz}, 2.5 \mathrm{GHz}$ and $5 \mathrm{GHz}$ for WLAN, WiFi and WiMax receivers. It also achieves similar good performance at these three different frequencies. The design is simulated and optimized in software Agilent Advanced Design System (ADS) and ANSOFT software . And we have demonstrated its good performance through other software Ansoft post simulation results. Such design for Cellular Transceiver in the Wireless applications is the first of its kind and the state-of-the-art performances distinguish the LNA in future multi-band applications. The tribande LNA exhibits input matching to $50 \mathrm{ohm}$ with $\mathrm{S} 11$ of $-9.34 \mathrm{~dB}$ at $1.9 \mathrm{GHz},-9.5 \mathrm{~dB}$ at $2.5 \mathrm{GHz}$ and $-23.5 \mathrm{~dB}$ at $5 . \mathrm{GHz}$ as well as output matching to $50 \mathrm{ohm}$ with $\mathrm{S}_{22}$ of $-5.5 \mathrm{~dB},-5.5 \mathrm{~dB}$ and $3.81 \mathrm{~dB}$, respectively. And it provides forward gain $\left(\mathrm{S}_{21}\right)$ of $29.3 \mathrm{~dB}, 24.56 \mathrm{~dB}$ and $11.93 \mathrm{~dB}$ with noise figure of $0.44 \mathrm{~dB}, 0.49 \mathrm{~dB}$ and $4.42 \mathrm{~dB}$ respectively while drawing $39 \mathrm{~mW}$ from a $5 \mathrm{~V}$ supply voltage.

\section{REFERENCES}

[1] Pozar D M. Microwave engineering. 3rd ed. NewYork: JWilley\& Sons, 2004

[2] Christina F. Jou, Kuo-Hua Cheng, Eing-Tsang Lu and Yang Wang, "Design Of A Fully Integrated Concurrent Triple-Band CMOS Low Noise Amplifier", IEEE, 2004

[3] F. Beaudoin et al., "A fully integrated tri-band, MIMO transceiver RFIC for 802.16e," in IEEE RFIC Symp. Dig., 2008, pp. 113-116.

[4] Chyuen-Wei Ang, Yuanjin Zheng, Chun-Huat Heng, “ A Multi-band CMOS Low Noise Amplifier for Multi-standard Wireless Receivers",ISCAS 2007 International Symposium on Circuits and Systems, 27 - 30 May 2007, 1-4244-0921-7/07 (C) 2007 IEEE, pp.2802-2805

[5] I. Akhchaf, S. Khoulji, M. Essaidi, M. L. Kerkeb, "Study, Modeling and Characterization of DualBand LNA Amplifiers Receivers for Wireless Microwaves Communication Systems" Journal of Wireless Networking and Communications 2012, 2(5): 77-82 DOI: 10.5923/j.jwnc.2012 02 05.01. 
[6] Kao, C.-Y., Y.-T. Chiang, and J.-R. Yang, "A concurrent multi-band low-noise ampli-er for WLAN/WiMAX applications," Proc.International Electro. Information Technology Conf., 514-517, 2008.

[7] Erick Emmanuel Djoumessi and Ke Wu, "Dual-Band Low-Noise Amplifier Using Step-Impedance Resonator (SIR) Technique for Wireless System Applications",Proceedings of the 39th European Microwave Conference; , Rome, Italy, 978-2-87487-011-8 EuMA 29 September, Page(s): 1307-1310 October 2009.

[8] K. Limet al.,"A 2x2 MIMO tri-band dual-mode CMOS transceiver for worldwide WiMAX/WLAN applications," in Proc. Eur. Solid-State Circuits Conf. (ESSCIRC), 2010, pp. 454-457.

[9] Phansathitwong, K., H. Sjoland, and P. Andreani, Low power multi-band CMOS receiver front-end," Proc. PRIME Conf., 1-4,2010

[10] Sambit Datta, Kunal Datta, Ashudeb Dutta, and Tarun Kanti Bhattacharyya, "Fully Concurrent DualBand LNA Operating in $900 \mathrm{MHz} / 2.4 \mathrm{GHz}$ Bands for Multi-Standard Wireless Receiver with sub2dB Noise Figure", ICETET, 3rd International Conference on Emerging Trends in Engineering and Technology, Page(s): 731-734, 2010.

[11] Runbo Ma and Wenmei Zhang, "Co design of Dual-band Low Noise Amplifier and Band-pass Filter", Asia-Pacific Symposium on Electromagnetic Compatibility (APEMC), IEEE, 2010, Page(s): 1378 1380, june 2010.

[12] Kai Xuan,1 Kim Fung Tsang, 1 and Wah Ching Lee2, "a novel tunable dual-band low noise amplifier for $868 / 915 \mathrm{mhz}$ and $2.4 \mathrm{ghz}$ zigbee application by cmos technology", Wiley Periodicals, Inc. Microwave and Optical Technology Letters, Vol. 52, No. 3, pages 507-510, March 2010.

[13] Sambit Datta, Kunal Datta, Ashudeb Dutta, and Tarun Kanti Bhattacharyya, "A Concurrent LowArea Dual Band 0.9/2.4 GHz LNA in $0.13 \mathrm{~m}$ RF CMOS Technology for Multi-Band Wireless Receiver", IEEE Asia Pacific Conference on Circuits and Systems (APCCAS), Page(s): 280 - 283, May 2010.

[14] Kyoohyun Lim ,"A 2x2 MIMO Tri-Band Dual-Mode Direct-Conversion CMOS Transceiver for Worldwide WiMAX/WLAN Applications", IEEE Journal of Solid-State Circuits, Vol. 46, No. 7 , Page(s): 1648 - 1658, July 2011.

[15] Zhe-Yang Huang1, and Chung-Chih Hung, "CMOS Dual-Band Low-Noise Amplifier for WorldWide WiMedia Ultra Wideband Wireless Personal Area Network System", Microwave Conference Proceedings (APMC), 2010 Asia-Pacific, Page(s): 334 - 337, March 2011.

[16] G.-L. Ning*, Z.-Y. Lei, L.-J. Zhang, R. Zou, and L. Shao, " Design of Concurrent low-noise amplifier for multi-band applications", Progress In Electromagnetics Research C, Vol. 22, 165-178, 2011

[17] Abhimanyu Athikayan, Aswathy Premanand, Athira Damodaran, Gayathry Girisan, " Design Of Low Noise Amplifier At 4 Ghz", 2011 International Conference on Information and Electronics Engineering, IPCSIT vol.6, 2011.

[18] Dong Min Kang and Hyung Sup Yoon "80-110 GHz MMIC amplifiers using a 0.1- m GaAs-based mHEMT technology", MICROWAVE AND OPTICAL TECHNOLOGY LETTERS, Volume 54, Issue 8, Pages: 1978-1982, August 2012.

[19] Shey-Shi Lu, Yo-Sheng Lin, Bo-Wei Lee, "Un monolithe 1.57/5.25-GHz double bande simultanée amplificateur à faible bruit en utilisant InGaP / GaAs HBT technologie", MICROWAVE AND OPTICAL TECHNOLOGY LETTERS, Volume 42, numéro 1, pages 58-60, 5 juillet 2004.

[20] Dario Benvenuti, Maurizio Cicolani, Stefano Pise, Pasquale Tommasino, Alessandro Trifiletti "Conception LNA large bande par FET en parallèle", MICROWAVE AND OPTICAL TECHNOLOGY LETTERS, Volume 32, numéro 4, pages 280-284, 20 février 2002. 


\section{Authors}

Ibtissam Akhchaf, was born in 1980 in Tetouan, Morocco, received his Master in Electronics and Telecommunication in 2008 at the University Abdelmalek Essaadi Tetouan. Currently, PhD student in the laboratory "Telecommunication Systems and Information" at the Faculty of Sciences of Tetouan. His research topic is especially RF circuits, microwave circuits and communications Mobiles.

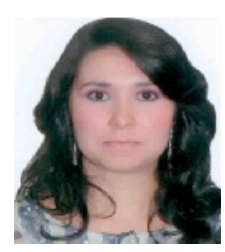

Samira Khoulji, teacher researcher at the Department of Statistics and Computer Science at the Faculty of Tetouan polydisciplinary (Morocco) and member of the research team "EMTI" Modeling and Information Theory. She received the PhD degree in Telecommunications in 2002 from the University Abdelmalek Essaadi, Tetouan, Morocco. His research on microwave circuits, RF systems, Wireless and Mobile Networks, mobile communications, wireless technology son, information technology and communication networks, sensors

Essaidi Mohammed, Director ENSIAS in Rabat (Morocco), was a Professor of Higher Education at the Faculty of Science at the University Abdelmalek Essadi, Tetouan, Morocco. He is Director of Research Laboratory "Telecommunication Systems and Information" and director of the department "and Microwave Electronics", responsible for several research and Moroccan as well as European head of IEEE Morocco. His main interests include the field of Telecommunication, microwave circuits and mobile technology.

Mohammed Larbi KERKEB, Professor of Higher Education at the Faculty of Physical Sciences, University Abdelmalek Essadi Tetouan, (Morocco). He is responsible for several research projects Moroccan and European. His main interests include modeling and design.
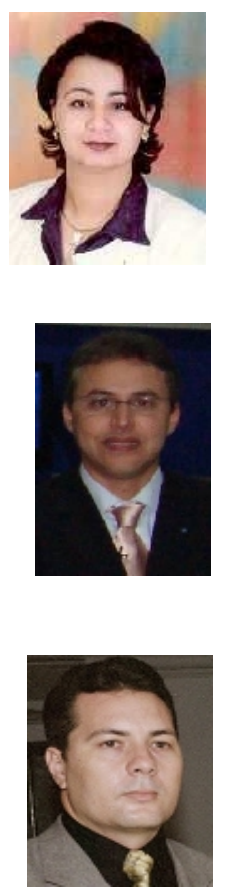